\title{
Article \\ Comparative Analysis of Lenvatinib and Hepatic Arterial Infusion Chemotherapy in Unresectable Hepatocellular Carcinoma: A Multi-Center, Propensity Score Study
}

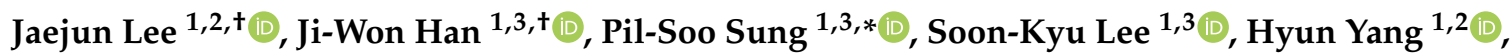 \\ Hee-Chul Nam ${ }^{1,4}$, Sun-Hong Yoo ${ }^{1,5}$, Hae-Lim Lee ${ }^{1,6}$, Hee-Yeon Kim ${ }^{1,4}{ }^{\mathbb{D}}$, Sung-Won Lee ${ }^{1,6}$, \\ Jung-Hyun Kwon 1,5, Jeong-Won Jang 1,3-1, Chang-Wook Kim ${ }^{1,4}{ }^{-1}$, Soon-Woo Nam ${ }^{1,5}$, Jung-Suk Oh ${ }^{7}$, \\ Ho-Jong Chun ${ }^{7}$, Si-Hyun Bae ${ }^{1,2}$, Jong-Young Choi ${ }^{1,3}$ and Seung-Kew Yoon ${ }^{1,3}$ (i)
}

Citation: Lee, J.; Han, J.-W.; Sung, P.-S.; Lee, S.-K.; Yang, H.; Nam, H.-C.; Yoo, S.-H.; Lee, H.-L.; Kim, H.-Y.; Lee, S.-W.; et al. Comparative Analysis of Lenvatinib and Hepatic Arterial Infusion Chemotherapy in Unresectable Hepatocellular Carcinoma: A Multi-Center, Propensity Score Study. J. Clin. Med. 2021, 10, 4045. https://doi.org/ $10.3390 / \mathrm{jcm} 10184045$

Academic Editor: Stanley W. Ashley

Received: 22 July 2021

Accepted: 4 September 2021

Published: 7 September 2021

Publisher's Note: MDPI stays neutral with regard to jurisdictional claims in published maps and institutional affiliations.

Copyright: (c) 2021 by the authors. Licensee MDPI, Basel, Switzerland. This article is an open access article distributed under the terms and conditions of the Creative Commons Attribution (CC BY) license (https:/ / creativecommons.org/licenses/by/ $4.0 /)$.
1 The Catholic University Liver Research Center, College of Medicine, The Catholic University of Korea, Seoul 06591, Korea; pwln0516@gmail.com (J.L.); tmznjf@catholic.ac.kr (J.-W.H.); blackiqq@gmail.com (S.-K.L.); oneggu@naver.com (H.Y.); hcnam128@catholic.ac.kr (H.-C.N.); calla0108@naver.com (S.-H.Y.); atom069@naver.com (H.-L.L.); hee82@catholic.ac.kr (H.-Y.K.); zambrotta@catholic.ac.kr (S.-W.L.); doctorkwon@catholic.ac.kr (J.-H.K.); garden@catholic.ac.kr (J.-W.J.); cwkim@catholic.ac.kr (C.-W.K.); drswnam@hanmail.net (S.-W.N.); baesh@catholic.ac.kr (S.-H.B.); jychoi@catholic.ac.kr (J.-Y.C.); yoonsk@catholic.ac.kr (S.-K.Y.)

2 Division of Gastroenterology and Hepatology, Department of Internal Medicine, College of Medicine, Eunpyeong St. Mary's Hospital, The Catholic University of Korea, Seoul 03382, Korea

3 Division of Gastroenterology and Hepatology, Department of Internal Medicine, College of Medicine, Seoul St. Mary's Hospital, The Catholic University of Korea, Seoul 06591, Korea

4 Division of Gastroenterology and Hepatology, Department of Internal Medicine, College of Medicine, Uijeongbu St. Mary's Hospital, The Catholic University of Korea, Seoul 11765, Korea

5 Division of Gastroenterology and Hepatology, Department of Internal Medicine, College of Medicine, Incheon St. Mary's Hospital, The Catholic University of Korea, Seoul 22711, Korea

6 Division of Gastroenterology and Hepatology, Department of Internal Medicine, College of Medicine, Bucheon St. Mary's Hospital, The Catholic University of Korea, Seoul 14647, Korea

7 Department of Radiology, College of Medicine, Seoul St. Mary's Hospital, The Catholic University of Korea, Seoul 06591, Korea; oj-cumc@hanmail.net (J.-S.O.); chunray@catholic.ac.kr (H.-J.C.)

* Correspondence: pssung@catholic.ac.kr; Tel.: +82-2258-2073; Fax: +82-2-3481-4025

+ These authors contribute equally to this work.

Abstract: The comparative efficacy and safety between lenvatinib and hepatic artery infusion chemotherapy (HAIC) in patients with unresectable hepatocellular carcinoma (HCC) is still unclear. This multicenter historical cohort study enrolled 244 patients who were treated with HAIC ( $n=173)$ or lenvatinib $(n=71)$ between 2012 and 2020. Propensity score matching (PSM) was performed, and 52 patients were selected per group. Clinical outcomes and safety were compared. Objective response rate (ORR) was not different between the two groups ( $26.0 \%$ vs. $23.1 \%, p=0.736)$. Before PSM, the HAIC group had a higher proportion of Child-Pugh B and portal vein tumor, whereas the lenvatinib group had more patients with extrahepatic metastases, which was adjusted after PSM. There were no differences in progression-free survival (PFS) and overall survival (OS) after PSM (HAIC vs. lenvatinib, median PFS, 3.6 vs. 4.0 months, $p=0.706$; median OS 10.8 vs. 7.9 months, $p=0.106)$. Multivariate Cox-regression showed that alpha-fetoprotein $\leq 1000 \mathrm{ng} / \mathrm{mL}$ was only an associated factor for OS after PSM in all patients (hazard ratio $=0.421, p=0.011$ ). Subgroup analysis for patients with a high tumor burden beyond the REFLECT eligibility criteria revealed that the HAIC group $(n=29)$ had a significantly longer OS than did the lenvatinib group ( $n=30)$ (10.0 vs. 5.4 months, $p=0.004$ ). More patients in the HAIC group achieved better liver function than those in the lenvatinib group at the time of best responses. There was no difference in the incidence of grade 3 and 4 adverse events between the two groups. Therefore, lenvatinib is comparable to HAIC in terms of ORR and OS in unresectable HCC meeting REFLECT eligibility criteria. 
Keywords: hepatocellular carcinoma; lenvatinib; hepatic arterial infusion chemotherapy; propensity score matching

\section{Introduction}

Hepatocellular carcinoma (HCC) is one of the most common causes of cancer-related deaths worldwide [1]. According to the Barcelona Clinic Liver Cancer (BCLC) staging system, HCC can be classified into five stages, and stage $C$ represents unresectable tumors with macrovascular invasion or extrahepatic spread [1]. For patients with BCLC stage $C$ or with BCLC-B who are not suitable for local treatments, systemic therapies have been recommended as the first-line therapy [2].

A recent randomized phase 3 trial showed that lenvatinib, a recently introduced tyrosine kinase inhibitor (TKI), is non-inferior to sorafenib in terms of overall survival (OS) in treatment-naïve unresectable HCC (the REFLECT trial) [3]. In addition, our group recently demonstrated that lenvatinib showed better progression-free survival (PFS) than sorafenib as a salvage treatment after transarterial treatment failure [4], which may be due to differences in molecular targets, including fibroblast growth factor pathways [5]. Nevertheless, the eligibility criteria for tumor burden in the REFLECT trial were only applicable to the selected patients (tumor extent $<50 \%$ of liver volume, absence of main portal vein tumor thrombosis (PVTT), absence of bile duct invasion) [6], and lenvatinib treatment in patients beyond these criteria showed varying outcomes, dependent on multiple factors, such as previous treatment history or PVTT [7].

Hepatic arterial infusion chemotherapy (HAIC) is considered a treatment option, instead of systemic chemotherapy, to reduce intrahepatic tumor burden in BCLC stage B or $\mathrm{C}$ with predominant intrahepatic disease by administering cytotoxic chemotherapeutic agents with high intrahepatic concentrations [8,9]. In addition, it can also be used in the poor responders of transarterial chemoembolization [10,11]. Therefore, HAIC is recommended as a therapeutic option for advanced HCC with vascular invasion in the Japanese guidelines [12]. Previous reports have shown that the control of intrahepatic tumors by HAIC provides survival benefits, even in patients with extrahepatic metastases or $\mathrm{Vp}$ $3 / 4$ PVTT $[13,14]$. Until now, there have been several studies comparing the efficacy of HAIC and sorafenib. A randomized phase 3 trial (SILIUS trial), which compared sorafenib alone to sorafenib plus HAIC, failed to show the superiority of the combination in advanced HCC [15]. However, more recently, another phase 3 randomized trial showed that oxaliplatin-based HAIC plus sorafenib exhibited a survival benefit compared to sorafenib alone in patients with HCC with portal vein invasion [16]. This implies that HAIC may be an effective treatment option in selected patient groups. Moreover, small prospective cohort studies showed that HAIC had a survival benefit compared with sorafenib in patients with advanced HCC with macrovascular invasion (MVI) without extrahepatic metastases $[17,18]$. Furthermore, a recent large-scale retrospective study using propensity score matching (PSM) analysis also reported that HAIC was superior to sorafenib in patients with advanced HCC with MVI without extrahepatic metastases in terms of OS [19]. However, no previous report has compared the real-world efficacy and safety between lenvatinib and HAIC in unresectable HCC.

Here, we performed a multicenter, historical cohort study in which HAIC and lenvatinib were compared in patients with unresectable HCC in terms of efficacy and safety. We used PSM to correct the various clinical parameters of patients with HCC, including tumor factors. We also analyzed the differences in survival outcomes between lenvatinib and HAIC in subgroup analysis with patients within or beyond the REFLECT eligibility criteria. 


\section{Patients and Methods}

\subsection{Study Population}

This study was approved by the Institutional Review Board of the Catholic University of Korea (approval number: XC21RIDI0008), and was performed in accordance with the Declaration of Helsinki. We retrospectively evaluated 244 consecutive patients with unresectable HCC who were treated with HAIC or lenvatinib at five affiliated hospitals in Korea. Patients treated with HAIC were enrolled from November 2012 to November 2020, whereas patients treated with lenvatinib were enrolled from January 2019 to November 2020. HCC was diagnosed by histological or radiological examinations via contrast-enhanced computed tomography (CT) and/or magnetic resonance imaging (MRI). The inclusion criteria were as follows: (1) confirmed intermediate to advanced HCC, but ineligible for surgical resection; (2) age $\geq 18$ years; and (3) Eastern Cooperative Eligibility criteria (ECOG) performance status score of $\leq 2$. The exclusion criteria were as follows: (1) lack of follow-up visits after the start of the treatment; (2) a treatment duration of $<2$ weeks for lenvatinibtreated patients; (3) less than two cycles of HAIC treatment for HAIC-treated patients; and (4) history of malignancy other than HCC in the previous 5 years.

\subsection{Treatment Protocol}

Lenvatinib was administered once daily at a dose of $8 \mathrm{mg}$ for patients weighing $<60 \mathrm{~kg}$, and at a dose of $12 \mathrm{mg}$ for patients weighing $>60 \mathrm{~kg}$. HAIC was performed as previously described $[18,20]$. The chemotherapy regimen consisted of 5-fluorouracil (5-FU) at a dose of $500 \mathrm{mg} / \mathrm{m}^{2} /$ day, and cisplatin at a dose of $60 \mathrm{mg} / \mathrm{m}^{2} /$ day. 5 -FU was administered for $5 \mathrm{~h}$ daily on days 1-3 and cisplatin for $2 \mathrm{~h}$ on day 1 or 2 . For arterial chemo-infusion, the catheter was inserted through the femoral artery and its tip was advanced to the common or proper hepatic artery; the other end of the tip was connected to the chemoport implanted in the subcutaneous pocket of the inguinal region. Each session was delivered every $3-4$ weeks via an implantable port system.

\subsection{Response Evaluation}

We classified the patients according to the BCLC stage, which was based on the radiologic and laboratory findings at the time of study enrollment. Imaging studies (CT or MRI) were conducted every 4-12 weeks for lenvatinib treatment and every 2-3 cycles of HAIC treatment for response evaluation. The assessment was conducted according to the modified Response Evaluation Criteria in Solid Tumors (mRECIST) as in our previous study [21]. OS was calculated from the start of drug administration until death or the last follow-up day. PFS was calculated as the time from the start of drug administration until disease progression, or drug cessation due to any cause in the absence of disease progression. The objective response rate (ORR) was calculated as the sum of the "complete response" and "partial response" at the response evaluation. The disease control rate (DCR) was calculated as the sum of the complete response (CR), partial response (PR), and stable disease (SD). The treatment response was defined as the best response during treatment. We categorized tumor types into nodular, massive, and diffuse types according to Eggel's classification [22]. Thereafter, massive and diffuse types were classified as non-nodular types. The modified albumin-bilirubin (mALBI) score was also measured to assess residual liver function at the end of each treatment as previously described [23], and with the following formula: $\mathrm{mALBI}=\left(\log _{10}\right.$ serum total bilirubin $\left.[\mu \mathrm{mol} / \mathrm{L}] \times 0.66\right)+($ serum albumin $[\mathrm{g} / \mathrm{L}]-0.085)$. Patients were divided into four groups according to the mALBI score: grade $1(<-2.60)$, grade $2 \mathrm{a}(2.60 \mathrm{ALBI}$ score $<-2.27)$, grade $2 \mathrm{~b}(-2.27)$, and grade $3(<-1.39)$. Adverse events were assessed according to the Common Terminology Criteria for Adverse Events version 4.0 [24].

\subsection{Propensity-Score Matching (PSM)}

We used PSM to adjust for differences in baseline characteristics between the HAIC $(n=173)$ and lenvatinib $(n=71)$ groups. Variables known to be related to the prognosis of HCC were selected for PSM, and included the ECOG, age, Child-Pugh score, extrahepatic 
metastasis and vascular invasion, intrahepatic tumor size, tumor type (nodular and nonnodular), and BCLC stage.

One-to-one nearest-neighbor matching within a caliper size of 0.20 was used. PSM analyses resulted in the selection of 52 patients in each group.

\subsection{Statistical Analysis}

All statistical analyses were performed using $\mathrm{R}$ statistical software (version 4.0.3; R Foundation Inc., Vienna, Austria; http: / / cran.r-project.org (access on 3 September 2021)) and SPSS version 23.0 software (SPSS, Chicago, IL, USA). The median clinical parameter values were calculated and the interquartile ranges were documented. The student's t-test was used to compare continuous variables between the two groups. The KaplanMeier method was used for survival analyses, including OS and PFS, and differences were examined using the log-rank test. Cox regression analyses were performed to identify the factors associated with survival outcomes, and factors with $p<0.01$ in univariate analysis were included in multivariate analysis. The therapeutic efficacy was demonstrated by the ORR and DCR, which were compared using the chi-square test. Statistical significance was defined as $p$-values $<0.05$.

\section{Results}

\subsection{Baseline Characteristics}

The baseline characteristics are presented in Table 1 . Among the 244 patients, 173 received HAIC and 71 received lenvatinib. The patients with HAIC were younger than those with lenvatinib (mean, 58.3 vs. 63.1 years old; $p=0.001$ ). In addition, the percentage of Child-Pugh B was higher in the HAIC group than in the lenvatinib group (52.6\% vs. $25.4 \%$, $p<0.001)$. The etiology was also significantly different between the two groups $(p=0.019)$; the HAIC group had a higher percentage of hepatitis B virus etiology $(77.5 \%$ vs. $59.2 \%)$ and a lower percentage of alcohol etiology (9.2\% vs. $22.5 \%)$. The median alpha-fetoprotein (AFP) level was higher (976 vs. $662.2 \mathrm{ng} / \mathrm{mL}, p=0.037$ ) in the HAIC group. The maximum tumor size was larger in the HAIC group (mean, 9.7 vs. $7.7 \mathrm{~cm} ; p=0.005$ ), and the nonnodular type was more frequent in the HAIC group $(63.0 \%$ vs. $28.2 \%, p<0.001)$, as was PVTT $(75.7 \%$ vs. $46.5 \%, p<0.001)$. In contrast, extrahepatic metastasis was more frequent in the lenvatinib group $(52.1 \%$ vs. $27.2 \%, p<0.001)$. A history of previous HCC treatment was more common in the lenvatinib group than in the HAIC group $(83.1 \%$ vs. $51.4 \%, p<0.001)$. Among those with a history of previous HCC treatment, 6 patients in the lenvatinib group and 12 patients in the HAIC group received either TKI or immune checkpoint inhibitor previously $(8.5 \%$ vs. $6.9 \%, p=0.681)$. Detailed previous treatment histories are presented in Table S1.

PSM was performed to adjust these differences in baseline characteristics between the two groups (Table 1), and 104 patients were selected for analysis after PSM (52 patients per group). No significant differences were observed between the two groups after PSM, except for median PIVKA-II levels $(p=0.046)$. 
Table 1. Baseline characteristics of the study population.

\begin{tabular}{|c|c|c|c|c|c|c|}
\hline \multicolumn{4}{|c|}{ Before PSM } & \multicolumn{3}{|c|}{ After PSM } \\
\hline Treatment & Lenvatinib $(n=71)$ & HAIC $(n=173)$ & $p$-Value & Lenvatinib $(n=52)$ & HAIC $(n=52)$ & $p$-Value \\
\hline Male sex & $62(87.3)$ & $150(86.7)$ & 1.000 & $47(90.4)$ & $45(86.5)$ & 0.760 \\
\hline Age (years) & $63.1 \pm 11.5$ & $58.3 \pm 10.2$ & 0.001 & $61.0 \pm 11.2$ & $61.2 \pm 11.6$ & 0.939 \\
\hline Child-Pugh & & & $<0.001$ & & & 1.000 \\
\hline A & $53(74.6)$ & $82(47.4)$ & & $34(65.4)$ & $33(63.5)$ & \\
\hline $\mathrm{B}$ & $18(25.4)$ & $91(52.6)$ & & $18(34.6)$ & $19(36.5)$ & \\
\hline Etiology & & & 0.019 & & & 0.585 \\
\hline HBV & $42(59.2)$ & $134(77.5)$ & & $32(61.5)$ & $37(71.2)$ & \\
\hline $\mathrm{HCV}$ & $7(9.9)$ & $13(7.5)$ & & $4(7.7)$ & $5(9.6)$ & \\
\hline Alcohol & $16(22.5)$ & $16(9.2)$ & & $12(23.1)$ & $8(15.4)$ & \\
\hline Others & $6(8.5)$ & $10(5.8)$ & & $4(7.7)$ & $2(3.8)$ & \\
\hline $\operatorname{AFP}(n g / m L)$ & $662.2(37.5-8000.2)$ & $\begin{array}{c}976 \\
(57.2-13,670)\end{array}$ & 0.037 & $1479.3(66.5-11,987)$ & $\begin{array}{c}308.51 \\
(29-12,979.5)\end{array}$ & 0.458 \\
\hline PIVKA (mAU/mL) & $\begin{array}{c}1648.5 \\
(107.9-20,154.9)\end{array}$ & $\begin{array}{c}1725 \\
(353-14,845)\end{array}$ & 0.673 & $\begin{array}{c}5850.5 \\
(130.8-25,629.3)\end{array}$ & $\begin{array}{c}872 \\
(405.5-4796.7)\end{array}$ & 0.046 \\
\hline Albumin (g/dL) & $3.7 \pm 0.5$ & $3.4 \pm 0.5$ & 0.001 & $3.5 \pm 0.5$ & $3.6 \pm 0.5$ & 0.527 \\
\hline Platelet $\left(10^{9} / \mathrm{L}\right)$ & $168.8 \pm 97.7$ & $174.9 \pm 104.7$ & 0.678 & $164.2 \pm 100.1$ & $176.0 \pm 105.8$ & 0.560 \\
\hline $\begin{array}{l}\text { Maximal tumor size } \\
\qquad(\mathrm{cm})\end{array}$ & $7.7 \pm 5.3$ & $9.7 \pm 4.8$ & 0.005 & $8.0 \pm 5.0$ & $8.1 \pm 4.8$ & 0.934 \\
\hline Tumor type & & & $<0.001$ & & & 0.549 \\
\hline Nodular & $51(71.8)$ & $64(37.0)$ & & $33(63.5)$ & $29(55.8)$ & \\
\hline Non-nodular & $20(28.2)$ & $109(63.0)$ & & $19(36.5)$ & $23(44.2)$ & \\
\hline PVTT & $33(46.5)$ & 131 (75.7) & $<0.001$ & $29(55.8)$ & $29(55.8)$ & 1.000 \\
\hline $\begin{array}{l}\text { Extrahepatic } \\
\text { metastasis }\end{array}$ & $37(52.1)$ & $47(27.2)$ & $<0.001$ & $20(38.5)$ & $24(46.2)$ & 0.552 \\
\hline BCLC & & & 0.408 & & & 0.625 \\
\hline B & $14(19.7)$ & $25(14.5)$ & & $12(23.1)$ & $9(17.3)$ & \\
\hline $\mathrm{C}$ & $57(80.3)$ & $148(85.5)$ & & $40(76.9)$ & $43(82.7)$ & \\
\hline ECOG & & & 0.160 & & & 0.578 \\
\hline 0 & $28(39.4)$ & $80(46.2)$ & & $19(36.5)$ & $18(34.6)$ & \\
\hline 1 & $39(54.9)$ & $74(42.8)$ & & $30(57.7)$ & $28(53.8)$ & \\
\hline 2 & $4(5.6)$ & $19(11.0)$ & & $3(5.8)$ & $6(11.5)$ & \\
\hline $\begin{array}{c}\text { Previous treatment } \\
\text { history }\end{array}$ & $59(83.1)$ & $89(51.4)$ & $<0.001$ & $40(76.9)$ & $30(57.7)$ & 0.060 \\
\hline
\end{tabular}

Data are presented as $n(\%)$, mean \pm SD, or median (IQR). PSM: propensity score matching, HAIC: hepatic arterial infusion chemotherapy, HBV: hepatitis B virus, HCV: hepatitis C virus, AFP: alpha-fetoprotein, PIVKA: protein induced by vitamin K antagonist, PVTT: portal vein tumor thrombosis, BCLC: Barcelona Clinic Liver Cancer, ECOG: Eastern Cooperative Oncology Group.

\subsection{Treatment Responses}

When we assessed treatment responses using the best response during treatment in the PSM cohort, $4(7.7 \%)$ patients in the HAIC group and $2(3.8 \%)$ patients in the lenvatinib group achieved CR, and $11(21.2 \%)$ patients in the HAIC group and $10(19.2 \%)$ patients in the lenvatinib group achieved PR. There was no statistical difference in ORR between the two groups (HAIC, $28.8 \%$ vs. lenvatinib, 23.1\%; $p=0.502$ ) (Table 2), although the DCR was different between the two groups $(73.1 \%$ in the HAIC group and $51.9 \%$ in the lenvatinib group; $p=0.026$ ), and especially the proportion of stable disease was higher in the HAIC group. These tendencies were also observed in the entire cohort without PSM (Table 2), which might be due to the high accumulation of chemotherapeutics within the tumor in HAIC. 
Table 2. Treatment responses before and after PSM.

\begin{tabular}{|c|c|c|c|c|c|c|}
\hline \multicolumn{4}{|c|}{ Before PSM } & \multicolumn{3}{|c|}{ After PSM } \\
\hline & Lenvatinib $(n=71)$ & HAIC $(n=173)$ & $p$-Value & Lenvatinib $(n=52)$ & HAIC $(n=52)$ & $p$-Value \\
\hline Treatment responses & & & 0.292 & & & 0.583 \\
\hline CR & $2(2.8)$ & $6(3.5)$ & & $2(3.8)$ & $4(7.7)$ & \\
\hline PR & $15(21.1)$ & $39(22.5)$ & & $10(19.2)$ & $11(21.2)$ & \\
\hline SD & $24(33.8)$ & $89(51.4)$ & & $15(28.8)$ & $23(44.2)$ & \\
\hline PD & $20(28.2)$ & $38(22.0)$ & & $16(30.8)$ & $13(25.0)$ & \\
\hline NA & $10(14.1)$ & $1(0.6)$ & & $9(17.3)$ & $1(1.9)$ & \\
\hline ORR & $17(23.9)$ & $45(26.0)$ & 0.736 & $12(23.1)$ & $15(28.8)$ & 0.502 \\
\hline DCR & $41(57.7)$ & $134(77.5)$ & 0.002 & 27 (51.9) & $38(73.1)$ & 0.026 \\
\hline
\end{tabular}

Data are presented as $n$ (\%). PSM: propensity score matching, HAIC: hepatic arterial infusion chemotherapy, CR: complete response, PR: partial response, SD: stable disease, PD: progressive disease, NA: not available, ORR: objective response rate, DCR: disease-controlled rate.

To assess early biological responses to treatment options, we analyzed the proportion of early AFP responses 4 weeks after the treatment, based on more than $20 \%$ or $50 \%$ reductions in AFP levels compared with the baseline levels, which are the most studied definitions [25]. As a result, the proportion of $\geq 20 \%$ AFP reduction at 4 weeks of treatment was not different between the lenvatinib and HAIC groups (Figure S1A). Similarly, the proportion of $\geq 50 \%$ AFP reduction at 4 weeks of treatment was also not significantly different between the two groups (Figure S1B). These findings are in line with the results of PFS, suggesting that early biological responses to lenvatinib might also be comparable to those of HAIC.

\subsection{Survival Outcomes}

We first compared the OS and PFS in the entire cohort without PSM. The median follow-up durations for the HAIC and lenvatinib groups were 6.9 and 4.8 months, respectively $(p<0.001)$, and the median treatment duration for HAIC and lenvatinib was 2.9 and 2.6 months, respectively $(p=0.159)$. The median OS was compared between the two groups, and no statistical difference was observed (HAIC, median of 9.4 months; $95 \%$ confidence interval [CI], 7.4-11.4 vs. lenvatinib, median of 9.3 months; $95 \% \mathrm{CI}, 6.8-11.8$; $p=0.489$ ) (Figure 1A). The median PFS was 3.7 months in the HAIC group (95\% CI, 3.0-4.5) and 4.3 months in the lenvatinib group ( $95 \% \mathrm{CI}, 2.9-5.7)$, with no statistical significance $(p=0.422)$ (Figure 1B).

After PSM, the median treatment duration did not differ significantly between the two groups (median of 2.9 months in the HAIC group and 2.5 months in the lenvatinib group; $p=0.150)$, either. In contrast, the median follow-up duration was significantly different (median of 7.7 months in the HAIC group and 4.2 months in the lenvatinib group; $p<0.001)$. During the follow-up period, $35(67.3 \%)$ patients in the lenvatinib group and $42(80.8 \%)$ patients in the HAIC group experienced disease progression or death. Although there was a tendency for longer OS in the HAIC group compared to the lenvatinib group (HAIC, median of 10.8 months, $95 \%$ CI, 6.9-14.8 vs. lenvatinib, median of 7.9 months, $95 \%$ CI, 4.2-11.7), the difference was not statistically significant ( $p=0.106$ ) (Figure 1C). Moreover, the PFS did not differ significantly between the two groups with median of 4.0 months (95\% CI, 2.5-5.5) in the lenvatinib group, and of 3.6 months $(95 \% \mathrm{CI}, 2.6-4.6)$ in the HAIC group $(p=0.706)$ (Figure 1D).

When we compared OS and PFS between lenvatinib and HAIC groups only in patients with non-viral HCC (Figure S2), there was no significant difference, which is consistent with analyses using the entire cohort, suggesting that our results may be applied regardless of etiology, although future studies are needed. 
A

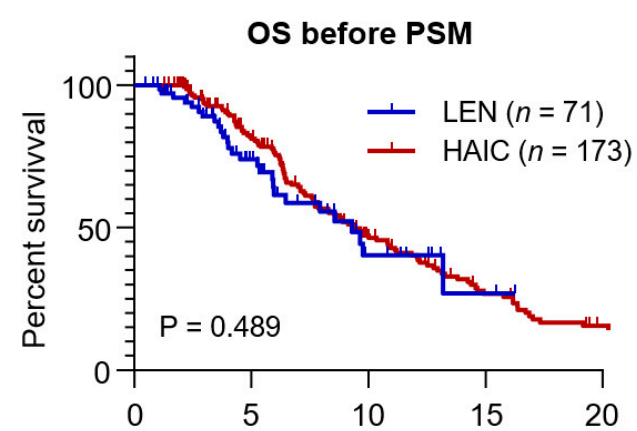

Number at risk

$\begin{array}{lccccc}\text { LEN } & 71 & 34 & 9 & 2 & 0\end{array}$

B

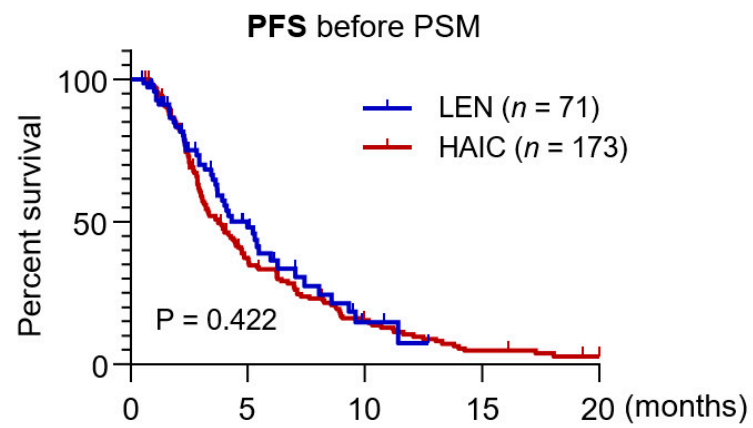

Number at risk

$\begin{array}{cccccc}\text { LEN } & 71 & 23 & 3 & 0 & 0 \\ \text { HAIC } & 173 & 55 & 19 & 6 & 2\end{array}$

D

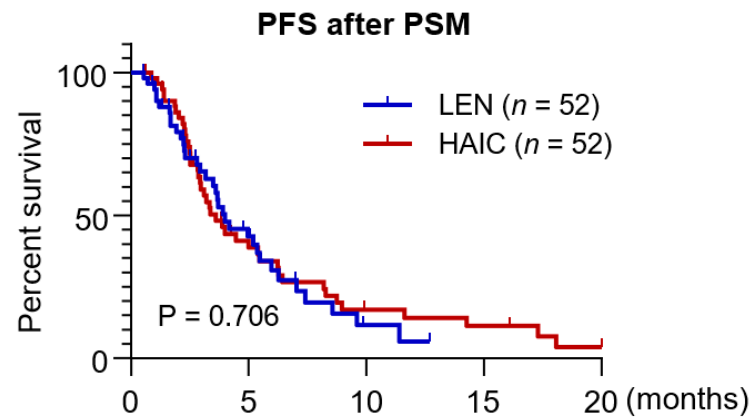

Number at risk

$\begin{array}{llllll}\text { LEN } & 52 & 16 & 2 & 0 & 0 \\ \text { HAIC } & 52 & 17 & 6 & 4 & 1\end{array}$

Figure 1. Kaplan-Meier survival curves analyzing OS and PFS in the HAIC and lenvatinib groups before $(n=244)$ and after PSM $(n=104)$. (A) Overall survival (OS) of patients treated with lenvatinib and HAIC in the entire cohort. (B) Progression free survival (PFS) of patients treated with lenvatinib and HAIC in the entire cohort. (C) OS of patients treated with lenvatinib and HAIC following propensity score matching (PSM). (D) PFS of patients treated with lenvatinib and HAIC following PSM. PSM: propensity score matching.

\subsection{Factors Contributing to Survival Outcomes}

Univariate and multivariate analyses were performed using the Cox proportional hazard model to identify factors associated with OS and PFS (Table 3) in the entire cohort. The cut-off value of AFP was determined to be $1000 \mathrm{ng} / \mathrm{mL}$ according to the analysis in our previous study [4]. In univariate analyses, age $>60$ years, intrahepatic maximal tumor size of $\leq 5 \mathrm{~cm}$, absence of extrahepatic metastasis, AFP levels $\leq 1000 \mathrm{ng} / \mathrm{mL}$, and Child-Pugh class A were factors associated with favorable OS. In multivariate analyses, extrahepatic metastasis (hazard ratio (HR), 1.6; 95\% CI, 1.1-2.3; $p=0.014$ ), Child-Pugh class A (HR, 0.7; 95\% CI, 0.5-0.9; $p=0.028$ ), and AFP levels $\leq 1000 \mathrm{ng} / \mathrm{mL}$ (HR, 0.7; 95\% CI, 0.5-0.9; $p=0.030$ ) were significant factors associated with OS. Regarding PFS, age $\leq 60$ years, intrahepatic maximal tumor size $\leq 5 \mathrm{~cm}$, extrahepatic metastasis, Child-Pugh class A, and AFP levels $\leq 1000 \mathrm{ng} / \mathrm{mL}$ were significant factors in univariate analyses. In multivariate analyses, Child-Pugh class A (HR, 0.7; 95\% CI, 0.5-0.9; $p=0.010)$ and AFP levels $\leq 1000 \mathrm{ng} / \mathrm{mL}$ (HR, $0.7 ; 95 \% \mathrm{CI}, 0.5-0.9 ; p=0.026$ ) were significant factors associated with PFS. 
Table 3. Univariate and multivariate analyses of the factors influencing OS and PFS in the entire cohort.

\begin{tabular}{|c|c|c|c|c|c|c|}
\hline \multirow[t]{2}{*}{ Variables } & \multicolumn{3}{|c|}{ Overall Survival } & \multicolumn{3}{|c|}{ Progression-Free Survival } \\
\hline & $\begin{array}{l}\text { Univariate } \\
\text { ( } p \text {-Value) }\end{array}$ & $\begin{array}{l}\text { Multivariate } \\
\text { ( } p \text {-Value) }\end{array}$ & HR $(95 \%$ CI) & $\begin{array}{l}\text { Univariate } \\
\text { ( } p \text {-Value) }\end{array}$ & $\begin{array}{c}\text { Multivariate } \\
(p \text {-Value) }\end{array}$ & HR $(95 \% \mathrm{CI})$ \\
\hline Lenvatinib vs. HAIC & 0.490 & & & 0.424 & & \\
\hline Age $\leq 60$ years & 0.005 & 0.098 & $1.4(0.9-1.9)$ & 0.010 & 0.105 & $1.3(0.9-1.7)$ \\
\hline HBV vs. non-HBV & 0.516 & & & 0.227 & & \\
\hline Tumor size $\leq 5 \mathrm{~cm}$ & 0.010 & 0.257 & $0.8(0.5-1.2)$ & 0.026 & 0.379 & $0.9(0.6-1.2)$ \\
\hline Macrovascular invasion & 0.211 & & & 0.108 & & \\
\hline Extrahepatic metastasis & 0.017 & 0.014 & $1.6(1.1-2.3)$ & 0.042 & 0.061 & $1.3(0.9-1.8)$ \\
\hline Child class A & 0.002 & 0.028 & $0.7(0.5-0.9)$ & $<0.001$ & 0.010 & $0.7(0.5-0.9)$ \\
\hline $\mathrm{AFP} \leq 1000$ & 0.001 & 0.030 & $0.7(0.5-0.9)$ & $<0.001$ & 0.026 & $0.7(0.5-0.9)$ \\
\hline PIVKA-II $\leq 1000$ & 0.067 & 0.353 & $0.8(0.6-1.2)$ & 0.137 & & \\
\hline
\end{tabular}

HAIC: hepatic arterial infusion chemotherapy, HBV: hepatitis B virus, AFP: alpha-fetoprotein, PIVKA-II: protein induced by vitamin K antagonist-II, HR: hazard ratio, CI: confidence interval.

Next, we performed subgroup analyses comparing OS between lenvatinib and HAIC groups according to the factors that could be associated with survival outcomes (Figure S3). Most subgroups did not show significant differences in HR between the two groups. However, patients in the lenvatinib group with macrovascular invasion $(\mathrm{HR}, 1.8 ; 95 \% \mathrm{CI}$, 1.0-3.0; $p=0.032$ ), maximal intrahepatic tumor size $>5 \mathrm{~cm}(\mathrm{HR}, 2.0 ; 95 \% \mathrm{CI}, 1.2-3.2$; $p=0.008)$, or AFP level > $1000 \mathrm{ng} / \mathrm{mL}(\mathrm{HR}, 1.8 ; 95 \% \mathrm{CI}, 1.0-3.1 ; p=0.034)$ showed inferior OS outcomes compared to the HAIC group. For PFS, the lenvatinib group showed better PFS than the HAIC group in patients with extrahepatic metastasis (HR, 0.5; $95 \% \mathrm{CI}, 0.3-0.8$; $p=0.003$ ) (Figure S4).

\subsection{Patients with Tumor Burden beyond the REFLECT Eligibility Criteria}

In the REFLECT trial, the eligibility criteria for tumor burden were strictly selected and comprised the following: Tumor extent $<50 \%$ of liver volume, absence of main PVTT, and absence of bile duct invasion. Patients with a tumor burden exceeding the REFLECT eligibility criteria were demonstrated as "REFLECT $(-)$," and those with a tumor burden within the REFLECT eligibility criteria were demonstrated as "REFLECT (+)." In the entire cohort, the ORR was not significantly different between the REFLECT ( + ) and REFLECT $(-)$ groups $(30.1 \%$ vs. $23.0 \%, p=0.225)$.

Figure 2 shows the Kaplan-Meier survival curve according to the REFLECT eligibility criteria and the type of treatment. In the entire cohort, REFLECT (+) patients showed better outcomes in survival rate compared to REFLECT $(-)$ patients (median of 14.6 vs. 7.7 months, $p<0.001$ ) (Figure 2A). Among REFLECT (-) patients, the HAIC group showed significantly higher OS than the lenvatinib group (median of 7.9 vs. 5.4 months, $p=0.003)$. When only considering patients after PSM, REFLECT $(+)$ patients also showed longer OS than REFLECT (-) PSM patients (median of 12.5 vs. 7.7 months, $p=0.006$ ). Furthermore, longer OS of the HAIC group compared to the lenvatinib group was also observed among REFLECT (-) PSM patients (median of 10.0 vs. 5.4 months, $p=0.004$ ) (Figure 2B). 


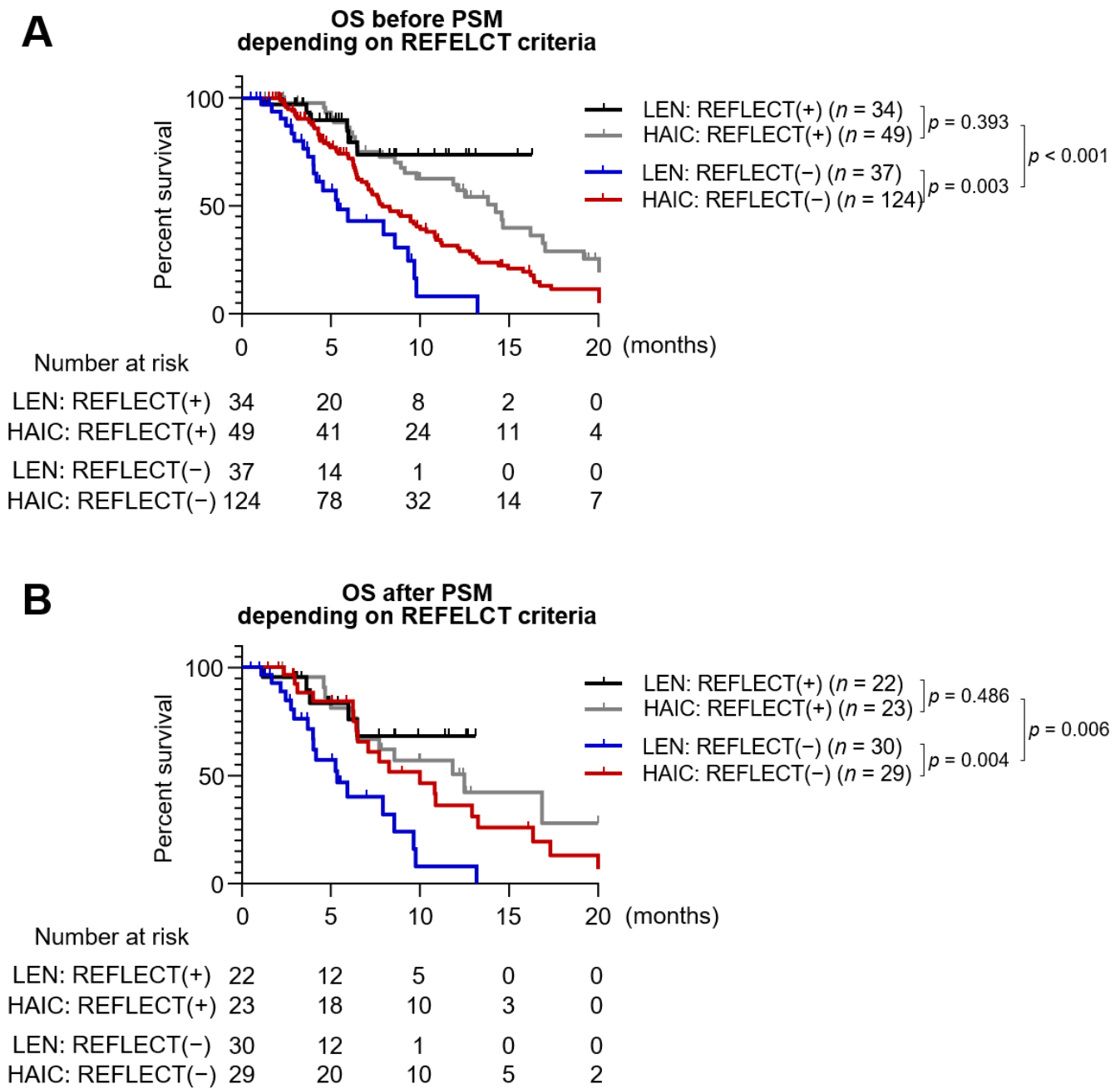

Figure 2. Kaplan-Meier survival curves analyzing OS according to the meeting the RELECT criteria and types of treatment. (A) OS in the entire cohort $(n=244)$. (B) OS in the PSM cohort $(n=104)$. REFLECT $(+)$ means "meeting the REFLECT criteria".

\subsection{Treatment-Related Toxicity}

Table 4 shows the adverse events, grade $\geq 3$ in the lenvatinib and HAIC groups after PSM. Elevation of aspartate aminotransferase was the most common severe adverse event $(11 / 52,21.2 \%)$ in the HAIC group, followed by the elevation of alanine aminotransferase $(7 / 52,13.5 \%)$ and hyperbilirubinemia $(7 / 52,13.5 \%)$. In the lenvatinib group, hypertension $(5 / 52,9.6 \%)$, thrombocytopenia $(5 / 52,9.6 \%)$, diarrhea $(5 / 52,9.6 \%)$ and hepatic encephalopathy $(5 / 52,9.6 \%)$ were the most common severe adverse events. Proteinuria was observed in three patients in the lenvatinib group. Overall, the prevalence of severe adverse events was not significantly different between the two groups, with $48.1 \%(25 / 52)$ in the HAIC group and $44.2 \%(23 / 52)$ in the lenvatinib group $(p=0.694)$. 
Table 4. Grade $\geq 3$ AEs associated with lenvatinib or HAIC treatment after PSM.

\begin{tabular}{|c|c|c|c|}
\hline Adverse Event & HAIC $(n=52)$ & Lenvatinib $(n=52)$ & $p$-Value \\
\hline AE grade $\geq 3$ (overlapped) & $25(48.1)$ & $23(44.2)$ & 0.694 \\
\hline HFSR & $0(0)$ & $2(3.8)$ & \\
\hline Hypertension & $0(0)$ & $5(9.6)$ & \\
\hline \multicolumn{4}{|l|}{ Nephrotoxicity } \\
\hline Proteinuria & $0(0)$ & $3(5.8)$ & \\
\hline Elevated creatinine & $2(3.8)$ & $0(0)$ & \\
\hline \multicolumn{4}{|l|}{ Hematologic } \\
\hline Anemia & $4(7.7)$ & $1(1.9)$ & \\
\hline Neutropenia & $1(1.9)$ & $0(0)$ & \\
\hline Thrombocytopenia & $2(3.8)$ & $5(9.6)$ & \\
\hline \multicolumn{4}{|l|}{ Laboratory } \\
\hline Hyperbilirubinemia & $7(13.5)$ & $3(5.8)$ & \\
\hline AST & $11(21.2)$ & $2(3.8)$ & \\
\hline ALT & $7(13.5)$ & $1(1.9)$ & \\
\hline \multicolumn{4}{|l|}{ Gastrointestinal } \\
\hline Nausea/vomiting & $3(5.8)$ & $2(3.8)$ & \\
\hline Diarrhea & $2(3.8)$ & $5(9.6)$ & \\
\hline Decreased appetite & $3(5.8)$ & $2(3.8)$ & \\
\hline Hepatic encephalopathy & $3(5.8)$ & $5(9.6)$ & \\
\hline Fatigue & $3(5.8)$ & $2(3.8)$ & \\
\hline Dyspnea & $0(0)$ & $1(1.9)$ & \\
\hline Abdominal pain & $1(1.9)$ & $0(0)$ & \\
\hline
\end{tabular}

The data are presented as $n(\%)$. AE: adverse event, HAIC: hepatic arterial infusion chemotherapy, HFSR: hand foot skin reaction, AST: aspartate aminotransferase, ALT: alanine transaminase.

\subsection{Liver Function after Lenvatinib or HAIC}

The residual liver function was evaluated using the Child-Pugh score and mALBI in each group following PSM (Table 5). Evaluation of residual liver function was done at the point of which the best treatment response was achieved. For those who had not undergone response evaluation, liver function was reviewed at one month after drug administration. As a result, $23(44.2 \%)$ patients in the lenvatinib group, and $35(67.3 \%)$ patients in the HAIC group showed Child-Pugh A liver function at the time of best responses, which was significantly higher in the HAIC group $(p=0.018)$. Furthermore, more patients in the HAIC group achieved better liver function by $\mathrm{mALBI} \leq 2 \mathrm{a}$ than those in the lenvatinib group $(48.1 \%$ vs. $25 \%$, respectively, $p=0.015)$ (Table 5). Overall, HAIC tended to preserve hepatic reserve compared to lenvatinib.

Table 5. Residual liver function at the time of best treatment response.

\begin{tabular}{cccc}
\hline & Lenvatinib $(\boldsymbol{n}=\mathbf{5 2})$ & HAIC $(\boldsymbol{n}=\mathbf{5 2})$ & $p$ \\
\hline Child-Pugh class A & $23(44.2)$ & $35(67.3)$ & 0.018 \\
mALBI grade $\leq 2 \mathrm{a}$ & $13(25.0)$ & $25(48.1)$ & 0.015 \\
\hline
\end{tabular}

The data are presented as $n(\%)$. Adm, Administration, mALBI, modified albumin-bilirubin.

Figure S5 shows the survival outcomes between groups with and without subsequent therapy in the PSM cohort. We only considered patients who discontinued the lenvatinib or HAIC therapies $(n=93)$. Forty patients who received lenvatinib or HAIC treatment received subsequent therapy and exhibited a median OS of 10.833 months, which was significantly longer than that of patients without subsequent therapy $(n=53$; median OS, 6.267 months; $p=0.033$ ). The number of patients who underwent subsequent therapy was significantly different between lenvatinib and HAIC groups $(p=0.015)$ (Table S2). Fourteen $(30.4 \%)$ patients in the lenvatinib group and $26(55.3 \%)$ in the HAIC group underwent subsequent therapy. Nivolumab $(n=4)$ was the most common choice for lenvatinib failure patients, whereas sorafenib $(n=11)$ was the most frequently selected drug for subsequent therapy after HAIC treatment. 


\section{Discussion}

To the best of our knowledge, this is the first report to compare the real-world outcomes of lenvatinib and HAIC in patients with unresectable HCC. There was no statistically significant difference in OS and PFS between the lenvatinib and HAIC groups before and after PSM. The REFLECT eligibility criteria included patients with a tumor extent $<50 \%$ of the liver volume, absence of main PVTT, and absence of bile duct invasion [3]. In patients beyond the REFLECT eligibility criteria, the HAIC group showed better OS than the lenvatinib group before and after PSM. There was no significant difference in severe adverse events between the two groups. These results suggest that lenvatinib and HAIC have similar efficacy and safety in unresectable HCC; however, selected groups with high intrahepatic tumor burden and PVTT may benefit from HAIC.

The REFLECT trial demonstrated that lenvatinib is not inferior to sorafenib as a firstline treatment in unresectable HCC in terms of OS (HR, 0.9; median of 13.6 months vs. 12.3 months) [3]. In this study, the REFLECT (+) group showed a mean OS of 14.6 months in the entire cohort and 12.5 months in the PSM cohort, which is consistent with the result from REFLECT trial. Regarding ORR, the total lenvatinib group in our study showed an ORR of 23.9\% (by mRECIST), which is compatible with recent real-world studies [4,26], and lower than that of the REFLECT trial. However, even for REFLECT (+) patients in this study, they showed ORR of $30.7 \%$ (by mRECIST), which is lower than that in REFLECT trial. [3] This may be due to the inclusion of a higher number of treatment-experienced patients in our study.

Regarding liver function following the treatment, lenvatinib significantly deteriorated liver function between baseline and week 2, and baseline to week 4, as measured by ALBI grade [27]. Preserved liver function at baseline in sequential treatment following TKI predicted improved prognosis [28], and early decline of liver function was associated with poor prognosis in unresectable HCC [27]. In agreement with this previous study, we observed a tendency for worsening of liver function in the lenvatinib group than in the HAIC group when showing best treatment responses (Child-Pugh A, $44.2 \%$ vs. $67.3 \%$; $p=0.018$, mALBI $\leq 2 \mathrm{a}, 25 \%$ vs. $48.1 \% ; p=0.015)$, resulting in a lower rate of inclusion following subsequent therapy in the Lenvatinib group ( $55.3 \%$ vs. $30.4 \%, p=0.015)$. We also showed that subsequent treatment following lenvatinib or HAIC was associated with longer OS, which emphasizes the importance of residual function following the treatments. Thus, HCC treatment in patients with poor liver function, such as Child-Pugh B in patients with advanced stage, remains a clinically unmet need. Although it is not generally recommended as a first-line treatment, HAIC is recommended as a therapeutic option for advanced HCC with vascular involvement, especially in the Japanese guidelines [12]. The Taiwanese and Korean guidelines suggest HAIC as an option for selected patients [29,30]. TKIs, including sorafenib or lenvatinib, are generally used in patients with Child-Pugh A, whereas HAIC can also be used in Child-Pugh B patients. A previous report suggested that HAIC does not significantly reduce liver function in Child-Pugh A patients [31]. Furthermore, HAIC improved liver function in responders when administered in Child-Pugh B patients, which might be linked to the resolution of vascular invasion [32,33]. Thus, HAIC may have clinical benefits in patients with poor liver function.

Previous studies have investigated the combination or sequential application of TKIs and HAIC. A recent randomized study, the SILIUS trial, compared sorafenib versus sorafenib plus HAIC in patients with unresectable HCC, including extrahepatic metastases [15]. Although there was no significant difference in OS between the groups, subgroup analyses showed that the combination treatment had a survival benefit in patients with main portal vein invasion [15], which is consistent with other previous reports [16,34]. Since lenvatinib seems to have a better tumor response rate than sorafenib, future studies are needed to identify the clinical benefit of the combination of lenvatinib and HAIC, especially in patients with vascular invasion. In this regard, a recent retrospective study showed that the lenvatinib, toriplimab, and HAIC combination regimen is superior to lenvatinib alone in terms of PFS and OS [35]. Furthermore, the survival benefit of sequential therapy, 
both TKI after HAIC failure or HAIC after TKI failure, remains unclear. A retrospective study showed that sorafenib treatment after HAIC failure had a higher survival rate than HAIC alone [36]. In contrast, HAIC improved survival after sorafenib failure [37], which demonstrated the potential role of sequential application of each treatment. Indeed, a previous study highlighted the effect of targeting intrahepatic lesions in prolonging survival following sorafenib treatment [38], and systemic therapies such as regorafenib and nivolumab showed poor responses rates following sorafenib treatment [39]. Therefore, future studies should investigate this sequential strategy in lenvatinib settings. Of note, continuing treatment following lenvatinib failure provided a survival benefit, suggesting that an effective post-progression treatment following lenvatinib failure still needs to be developed [40].

Despite the retrospective design, our study is the first to show comparable clinical outcomes of lenvatinib and HAIC in patients with unresectable HCC, although HAIC had better survival outcomes in selected patient groups such as patients beyond REFLECT criteria. Future large-scale, prospective studies are needed to validate our results.

Supplementary Materials: The following are available online at https:/ / www.mdpi.com/article/ $10.3390 / \mathrm{jcm} 10184045 / \mathrm{s} 1$, Figure S1: Graphs comparing early AFP response ( $\geq 20 \%$ reduction of AFP at 4 weeks of treatment (A), and $\geq 50 \%$ reduction of AFP at 4 weeks of treatment (B), in each treatment group; Figure S2: Kaplan-Meier curves of non-viral HCC comparing lenvatinib and HAIC before and after PSM; Figure S3: Forest plot comparing lenvatinib and HAIC group which representing hazard ratio, $p$-value, and median OS in each subgroup; Figure S4: Forest plot comparing lenvatinib and HAIC group which representing hazard ratio, $p$-value, and median PFS in each subgroup; Figure S5: Kaplan-Meier curve representing survival difference between patients with salvage therapy and without salvage therapy at the end of HAIC or lenvatinib treatment, in PSM cohort; Table S1: Previous treatment history before lenvatinib or HAIC treatment classified according to the BCLC stage; Table S2: Salvage therapy following lenvatinib or HAIC.

Author Contributions: P.-S.S.: study design, data collection, data analysis, data interpretation, manuscript writing, and manuscript approval. J.L. and J.-W.H.: study design, data collection, data analysis, data interpretation, and manuscript writing. H.Y., S.-K.L., H.-C.N., S.-H.Y., H.-L.L., H.-Y.K., S.-W.L.: data collection. J.-H.K., J.-W.J., C.-W.K., S.-W.N., J.-S.O., H.-J.C., S.-H.B., J.-Y.C., S.-K.Y.: data interpretation and manuscript approval. All authors have read and agreed to the published version of the manuscript.

Funding: This research was supported by the Basic Science Research Program through a National Research Foundation of Korea (NRF) grant funded by the Korean government (MSIT) (2021R1C1C1005844 to P.S.S.). This research was also supported by the Basic Science Research Program through a NRF grant funded by the Ministry of Education (NRF-2019R1I1A1A01059642 to P.S.S.). This study was also supported by the Research Fund of Seoul St. Mary's Hospital, The Catholic University of Korea (to J.W.H.). This study was also supported by The Catholic University of Korea, Eunpyeong St. Mary's Hospital, Research Institute of Medical Science (to H.Y.).

Institutional Review Board Statement: The study was conducted according to the guidelines of the Declaration of Helsinki, and approved by the Institutional Review Board of the Catholic University of Korea (approval number: XC21RIDI0008).

Informed Consent Statement: Informed consent was not required, because this research was retrospective study.

Data Availability Statement: Data is contained within the article.

Conflicts of Interest: The authors declare no conflict of interest. The funders had no role in the design of the study; in the collection, analyses, or interpretation of data; in the writing of the manuscript, or in the decision to publish the results. The funders had no role in the design of the study; in the collection, analyses, or interpretation of data; in the writing of the manuscript, or in the decision to publish the results. 


\section{References}

1. Villanueva, A. Hepatocellular Carcinoma. N. Engl. J. Med. 2019, 380, 1450-1462. [CrossRef]

2. European Association for the Study of the Liver; European Organisation for Research and Treatment of Cancer. EASL-EORTC clinical practice guidelines: Management of hepatocellular carcinoma. J. Hepatol. 2012, 56, 908-943. [CrossRef] [PubMed]

3. Kudo, M.; Finn, R.S.; Qin, S.; Han, K.H.; Ikeda, K.; Piscaglia, F.; Baron, A.; Park, J.W.; Han, G.; Jassem, J.; et al. Lenvatinib versus sorafenib in first-line treatment of patients with unresectable hepatocellular carcinoma: A randomised phase 3 non-inferiority trial. Lancet 2018, 391, 1163-1173. [CrossRef]

4. Lee, J.; Sung, P.S.; Yang, H.; Lee, S.K.; Nam, H.C.; Yoo, S.H.; Lee, H.L.; Kim, H.Y.; Lee, S.W.; Kwon, J.H.; et al. A Real-World Comparative Analysis of Lenvatinib and Sorafenib as a Salvage Therapy for Transarterial Treatments in Unresectable HCC. J. Clin. Med. 2020, 9, 4121. [CrossRef] [PubMed]

5. Hoshi, T.; Watanabe Miyano, S.; Watanabe, H.; Sonobe, R.M.K.; Seki, Y.; Ohta, E.; Nomoto, K.; Matsui, J.; Funahashi, Y. Lenvatinib induces death of human hepatocellular carcinoma cells harboring an activated FGF signaling pathway through inhibition of FGFR-MAPK cascades. Biochem. Biophys. Res. Commun. 2019, 513, 1-7. [CrossRef] [PubMed]

6. Duran, S.R.; Jaquiss, R.D.B. Hepatocellular Carcinoma. N. Engl. J. Med. 2019, 381, e2. [CrossRef] [PubMed]

7. Sho, T.; Suda, G.; Ogawa, K.; Shigesawa, T.; Suzuki, K.; Nakamura, A.; Ohara, M.; Umemura, M.; Kawagishi, N.; Natsuizaka, M.; et al. Lenvatinib in patients with unresectable hepatocellular carcinoma who do not meet the REFLECT trial eligibility criteria. Hepatol. Res. 2020, 50, 966-977. [CrossRef]

8. Kudo, M.; Matsui, O.; Izumi, N.; Iijima, H.; Kadoya, M.; Imai, Y.; Okusaka, T.; Miyayama, S.; Tsuchiya, K.; Ueshima, K.; et al. JSH Consensus-Based Clinical Practice Guidelines for the Management of Hepatocellular Carcinoma: 2014 Update by the Liver Cancer Study Group of Japan. Liver Cancer 2014, 3, 458-468. [CrossRef]

9. $\quad$ Sung, P.S.; Choi, M.H.; Yang, H.; Lee, S.K.; Chun, H.J.; Jang, J.W.; Choi, J.Y.; Yoon, S.K.; Choi, J.I.; Lee, Y.J.; et al. Diffusion-Weighted Magnetic Resonance Imaging in Hepatocellular Carcinoma as a Predictor of a Response to Cisplatin-Based Hepatic Arterial Infusion Chemotherapy. Front. Oncol. 2020, 10, 600233. [CrossRef]

10. Lee, J.S.; Kim, B.K.; Kim, S.U.; Park, J.Y.; Ahn, S.H.; Seong, J.S.; Han, K.H.; Kim, D.Y. A survey on transarterial chemoembolization refractoriness and a real-world treatment pattern for hepatocellular carcinoma in Korea. Clin. Mol. Hepatol. 2020, $26,24-32$. [CrossRef]

11. Moriya, K.; Namisaki, T.; Sato, S.; Furukawa, M.; Douhara, A.; Kawaratani, H.; Kaji, K.; Shimozato, N.; Sawada, Y.; Saikawa, S.; et al. Bi-monthly hepatic arterial infusion chemotherapy as a novel strategy for advanced hepatocellular carcinoma in decompensated cirrhotic patients. Clin. Mol. Hepatol. 2019, 25, 381-389. [CrossRef]

12. Kokudo, N.; Takemura, N.; Hasegawa, K.; Takayama, T.; Kubo, S.; Shimada, M.; Nagano, H.; Hatano, E.; Izumi, N.; Kaneko, S.; et al. Clinical practice guidelines for hepatocellular carcinoma: The Japan Society of Hepatology 2017 (4th JSH-HCC guidelines) 2019 update. Hepatol. Res. 2019, 49, 1109-1113. [CrossRef]

13. Sung, P.S.; Yang, K.; Bae, S.H.; Oh, J.S.; Chun, H.J.; Nam, H.C.; Jang, J.W.; Choi, J.Y.; Yoon, S.K. Reduction of Intrahepatic Tumour by Hepatic Arterial Infusion Chemotherapy Prolongs Survival in Hepatocellular Carcinoma. Anticancer Res. 2019, 39, 3909-3916. [CrossRef]

14. Lin, C.C.; Hung, C.F.; Chen, W.T.; Lin, S.M. Hepatic Arterial Infusion Chemotherapy for Advanced Hepatocellular Carcinoma with Portal Vein Thrombosis: Impact of Early Response to 4 Weeks of Treatment. Liver Cancer 2015, 4, 228-240. [CrossRef]

15. Kudo, M.; Ueshima, K.; Yokosuka, O.; Ogasawara, S.; Obi, S.; Izumi, N.; Aikata, H.; Nagano, H.; Hatano, E.; Sasaki, Y.; et al. Sorafenib plus low-dose cisplatin and fluorouracil hepatic arterial infusion chemotherapy versus sorafenib alone in patients with advanced hepatocellular carcinoma (SILIUS): A randomised, open label, phase 3 trial. Lancet Gastroenterol. Hepatol. 2018, 3 , 424-432. [CrossRef]

16. He, M.; Li, Q.; Zou, R.; Shen, J.; Fang, W.; Tan, G.; Zhou, Y.; Wu, X.; Xu, L.; Wei, W.; et al. Sorafenib Plus Hepatic Arterial Infusion of Oxaliplatin, Fluorouracil, and Leucovorin vs Sorafenib Alone for Hepatocellular Carcinoma With Portal Vein Invasion: A Randomized Clinical Trial. JAMA Oncol. 2019, 5, 953-960. [CrossRef]

17. Nakano, M.; Niizeki, T.; Nagamatsu, H.; Tanaka, M.; Kuromatsu, R.; Satani, M.; Okamura, S.; Iwamoto, H.; Shimose, S.; Shirono, T.; et al. Clinical effects and safety of intra-arterial infusion therapy of cisplatin suspension in lipiodol combined with 5-fluorouracil versus sorafenib, for advanced hepatocellular carcinoma with macroscopic vascular invasion without extra-hepatic spread: A prospective cohort study. Mol. Clin. Oncol. 2017, 7, 1013-1020. [CrossRef] [PubMed]

18. Choi, J.H.; Chung, W.J.; Bae, S.H.; Song, D.S.; Song, M.J.; Kim, Y.S.; Yim, H.J.; Jung, Y.K.; Suh, S.J.; Park, J.Y.; et al. Randomized, prospective, comparative study on the effects and safety of sorafenib vs. hepatic arterial infusion chemotherapy in patients with advanced hepatocellular carcinoma with portal vein tumor thrombosis. Cancer Chemother. Pharmacol. 2018, 82, 469-478. [CrossRef] [PubMed]

19. Iwamoto, H.; Niizeki, T.; Nagamatsu, H.; Ueshima, K.; Nomura, T.; Kuzuya, T.; Kasai, K.; Kooka, Y.; Hiraoka, A.; Sugimoto, R.; et al. Survival Benefit of Hepatic Arterial Infusion Chemotherapy over Sorafenib in the Treatment of Locally Progressed Hepatocellular Carcinoma. Cancers 2021, 13, 646. [CrossRef]

20. Song, D.S.; Song, M.J.; Bae, S.H.; Chung, W.J.; Jang, J.Y.; Kim, Y.S.; Lee, S.H.; Park, J.Y.; Yim, H.J.; Cho, S.B.; et al. A comparative study between sorafenib and hepatic arterial infusion chemotherapy for advanced hepatocellular carcinoma with portal vein tumor thrombosis. J. Gastroenterol. 2015, 50, 445-454. [CrossRef] 
21. Sung, P.S.; Park, H.L.; Yang, K.; Hwang, S.; Song, M.J.; Jang, J.W.; Choi, J.Y.; Yoon, S.K.; Yoo, I.R.; Bae, S.H. (18)F-fluorodeoxyglucose uptake of hepatocellular carcinoma as a prognostic predictor in patients with sorafenib treatment. Eur. J. Nucl. Med. Mol. Imaging 2018, 45, 384-391. [CrossRef]

22. Rhee, H.; Chung, T.; Yoo, J.E.; Nahm, J.H.; Woo, H.Y.; Choi, G.H.; Han, D.H.; Park, Y.N. Gross type of hepatocellular carcinoma reflects the tumor hypoxia, fibrosis, and stemness-related marker expression. Hepatol. Int. 2020, 14, 239-248. [CrossRef] [PubMed]

23. Hiraoka, A.; Kumada, T.; Tsuji, K.; Takaguchi, K.; Itobayashi, E.; Kariyama, K.; Ochi, H.; Tajiri, K.; Hirooka, M.; Shimada, N.; et al. Validation of Modified ALBI Grade for More Detailed Assessment of Hepatic Function in Hepatocellular Carcinoma Patients: A Multicenter Analysis. Liver Cancer 2019, 8, 121-129. [CrossRef] [PubMed]

24. Dueck, A.C.; Mendoza, T.R.; Mitchell, S.A.; Reeve, B.B.; Castro, K.M.; Rogak, L.J.; Atkinson, T.M.; Bennett, A.V.; Denicoff, A.M.; O'Mara, A.M.; et al. Validity and Reliability of the US National Cancer Institute's Patient-Reported Outcomes Version of the Common Terminology Criteria for Adverse Events (PRO-CTCAE). JAMA Oncol. 2015, 1, 1051-1059. [CrossRef] [PubMed]

25. He, C.; Peng, W.; Liu, X.; Li, C.; Li, X.; Wen, T.F. Post-treatment alpha-fetoprotein response predicts prognosis of patients with hepatocellular carcinoma: A meta-analysis. Medicine (Baltimore) 2019, 98, e16557. [CrossRef] [PubMed]

26. Goh, M.J.; Oh, J.H.; Park, Y.; Kim, J.; Kang, W.; Sinn, D.H.; Gwak, G.Y.; Paik, Y.H.; Choi, M.S.; Lee, J.H.; et al. Efficacy and Safety of Lenvatinib Therapy for Unresectable Hepatocellular Carcinoma in a Real-World Practice in Korea. Liver Cancer 2021, 10, 52-62. [CrossRef]

27. Hiraoka, A.; Kumada, T.; Atsukawa, M.; Hirooka, M.; Tsuji, K.; Ishikawa, T.; Takaguchi, K.; Kariyama, K.; Itobayashi, E.; Tajiri, K.; et al. Early Relative Change in Hepatic Function with Lenvatinib for Unresectable Hepatocellular Carcinoma. Oncology 2019, 97, 334-340. [CrossRef]

28. Hiraoka, A.; Kumada, T.; Atsukawa, M.; Hirooka, M.; Tsuji, K.; Ishikawa, T.; Takaguchi, K.; Kariyama, K.; Itobayashi, E.; Tajiri, K.; et al. Important Clinical Factors in Sequential Therapy Including Lenvatinib against Unresectable Hepatocellular Carcinoma. Oncology 2019, 97, 277-285. [CrossRef]

29. Korean Liver Cancer, A.; National Cancer, C. 2018 Korean Liver Cancer Association-National Cancer Center Korea Practice Guidelines for the Management of Hepatocellular Carcinoma. Gut Liver 2019, 13, 227-299. [CrossRef]

30. Surveillance Group; Diagnosis Group; Staging Group; Surgery Group; Local ablation Group; TACE/TARE/HAI Group; Target therapy/systemic therapy Group; Radiotherapy Group; Prevention Group; Drafting Group; et al. Management consensus guideline for hepatocellular carcinoma: 2016 updated by the Taiwan Liver Cancer Association and the Gastroenterological Society of Taiwan. J. Formos. Med. Assoc. 2018, 117, 381-403. [CrossRef]

31. Terashima, T.; Yamashita, T.; Arai, K.; Kawaguchi, K.; Kitamura, K.; Yamashita, T.; Sakai, Y.; Mizukoshi, E.; Honda, M.; Kaneko, S. Beneficial Effect of Maintaining Hepatic Reserve during Chemotherapy on the Outcomes of Patients with Hepatocellular Carcinoma. Liver Cancer 2017, 6, 236-249. [CrossRef]

32. Terashima, T.; Yamashita, T.; Arai, K.; Kawaguchi, K.; Kitamura, K.; Yamashita, T.; Sakai, Y.; Mizukoshi, E.; Honda, M.; Kaneko, S. Response to chemotherapy improves hepatic reserve for patients with hepatocellular carcinoma and Child-Pugh B cirrhosis. Cancer Sci. 2016, 107, 1263-1269. [CrossRef] [PubMed]

33. Saeki, I.; Yamasaki, T.; Maeda, M.; Hisanaga, T.; Iwamoto, T.; Matsumoto, T.; Hidaka, I.; Ishikawa, T.; Takami, T.; Sakaida, I. Evaluation of the "assessment for continuous treatment with hepatic arterial infusion chemotherapy" scoring system in patients with advanced hepatocellular carcinoma. Hepatol. Res. 2018, 48, E87-E97. [CrossRef] [PubMed]

34. Ikeda, M.; Shimizu, S.; Sato, T.; Morimoto, M.; Kojima, Y.; Inaba, Y.; Hagihara, A.; Kudo, M.; Nakamori, S.; Kaneko, S.; et al. Sorafenib plus hepatic arterial infusion chemotherapy with cisplatin versus sorafenib for advanced hepatocellular carcinoma: Randomized phase II trial. Ann. Oncol. 2016, 27, 2090-2096. [CrossRef] [PubMed]

35. He, M.-K.; Liang, R.-B.; Zhao, Y.; Xu, Y.-J.; Chen, H.-W.; Zhou, Y.-M.; Lai, Z.-C.; Xu, L.; Wei, W.; Zhang, Y.-J. Lenvatinib, toripalimab, plus hepatic arterial infusion chemotherapy versus lenvatinib alone for advanced hepatocellular carcinoma. Ther. Adv. Med. Oncol. 2021, 13, 17588359211002720. [CrossRef]

36. Miyaki, D.; Aikata, H.; Kan, H.; Fujino, H.; Urabe, A.; Masaki, K.; Fukuhara, T.; Kobayashi, T.; Naeshiro, N.; Nakahara, T.; et al. Clinical outcome of sorafenib treatment in patients with advanced hepatocellular carcinoma refractory to hepatic arterial infusion chemotherapy. J. Gastroenterol. Hepatol. 2013, 28, 1834-1841. [CrossRef]

37. Kondo, M.; Numata, K.; Hara, K.; Nozaki, A.; Fukuda, H.; Chuma, M.; Maeda, S.; Tanaka, K. Treatment of Advanced Hepatocellular Carcinoma after Failure of Sorafenib Treatment: Subsequent or Additional Treatment Interventions Contribute to Prolonged Survival Postprogression. Gastroenterol. Res. Pract. 2017, 2017, 5728946. [CrossRef]

38. Terashima, T.; Yamashita, T.; Horii, R.; Arai, K.; Kawaguchi, K.; Kitamura, K.; Yamashita, T.; Sakai, Y.; Mizukoshi, E.; Honda, M.; et al. Potential efficacy of therapies targeting intrahepatic lesions after sorafenib treatment of patients with hepatocellular carcinoma. BMC Cancer 2016, 16, 338. [CrossRef]

39. Lee, C.H.; Lee, Y.B.; Kim, M.A.; Jang, H.; Oh, H.; Kim, S.W.; Cho, E.J.; Lee, K.H.; Lee, J.H.; Yu, S.J.; et al. Effectiveness of nivolumab versus regorafenib in hepatocellular carcinoma patients who failed sorafenib treatment. Clin. Mol. Hepatol. 2020, 26, 328-339. [CrossRef]

40. Hiraoka, A.; Kumada, T.; Tada, T.; Kariyama, K.; Tani, J.; Fukunishi, S.; Atsukawa, M.; Hirooka, M.; Tsuji, K.; Ishikawa, T.; et al. What Can Be Done to Solve the Unmet Clinical Need of Hepatocellular Carcinoma Patients following Lenvatinib Failure? Liver Cancer 2021. [CrossRef] 